\title{
Assessment of rotatory laxity in anterior cruciate ligament- deficient knees using magnetic resonance imaging with Porto-knee testing device
}

\author{
João Espregueira-Mendes · Hélder Pereira • Nuno Sevivas • \\ Cláudia Passos • José C. Vasconcelos • Alberto Monteiro • \\ Joaquim M. Oliveira • Rui L. Reis
}

Received: 9 December 2011 / Accepted: 18 January 2012/Published online: 31 January 2012

(C) Springer-Verlag 2012

\begin{abstract}
Purpose Objective evaluation of both antero-posterior translation and rotatory laxity of the knee remains a target to be accomplished. This is true for both preoperative planning and postoperative assessment of different ACL reconstruction emerging techniques. The ideal measurement tool should be simple, accurate and reproducible, while enabling to assess both "anatomy" and "function" during the same examination. The purpose of this study is to evaluate the clinical effectiveness of a new in-housedeveloped testing device, the so-called Porto-knee testing device (PKTD). The PKTD is aimed to be used on the
\end{abstract}

J. Espregueira-Mendes $(\bowtie) \cdot$ H. Pereira $\cdot$ N. Sevivas .

C. Passos · J. C. Vasconcelos - A. Monteiro

Saúde Atlântica Sports Center-F.C. Porto Stadium,

Minho University and Porto University, Porto, Portugal

e-mail: jem@espregueira.com

J. Espregueira-Mendes - H. Pereira - J. M. Oliveira - R. L. Reis

3B's Research Group-Biomaterials, Biodegradables and Biomimetics, Headquarters of the European Institute of Excellence on Tissue Engineering and Regenerative Medicine, University of Minho, AvePark, S. Cláudio de Barco, 4806-909 Taipas, Guimarães, Portugal

J. Espregueira-Mendes $\cdot$ H. Pereira $\cdot$ N. Sevivas $\cdot$

J. M. Oliveira $\cdot$ R. L. Reis

ICVS/3B's—PT Government Associate Laboratory,

Braga/Guimarães, Portugal

H. Pereira

Orthopedic Department,

Centro Hospitalar Póvoa de Varzim,

Vila do Conde, Portugal

N. Sevivas

Orthopedic Department, Hospital de Braga,

Braga, Portugal evaluation of both antero-posterior and rotatory laxity of the knee during MRI exams.

Methods Between 2008 and 2010, 33 patients with ACLdeficient knees were enrolled for the purpose of this study. All patients were evaluated in the office and under anesthesia with Lachman test, lateral pivot-shift test and anterior drawer test. All cases were studied preoperatively with KT-1000 and MRI with PKTD, and examinations performed by independent observers blinded for clinical evaluation. During MRI, we have used a PKTD that applies antero-posterior translation and permits free tibial rotation through a standardized pressure $(46.7 \mathrm{kPa})$ in the proximal posterior region of the leg. Measurements were taken for both knees and comparing side-to-side. Five patients with partial ruptures were excluded from the group of 33 .

Results For the 28 remaining patients, 3 women and 25 men, with mean age of $33.4 \pm 9.4$ years, 13 left and 15 right knees were tested. No significant correlation was noticed for Lachman test and PKTD results (n.s.). Pivot-shift had a strong positive correlation with the difference in anterior translation registered in lateral and medial tibia plateaus of injured knees (cor. coefficient $=0.80 ; p<0.05$ ), and with the difference in this parameter as compared to side-to-side (cor. coefficient $=0.83 ; p<0.05$ ).

Considering the KT-1000 difference between injured and healthy knees, a very strong positive correlation was found for side-to-side difference in medial (cor. coefficient $=0.73 ; p<0.05$ ) and lateral (cor. coefficient $=0.5$; $p<0.05)$ tibial plateau displacement using PKTD.

Conclusion The PKTD proved to be a reliable tool in assessment of antero-posterior translation (comparing with KT-1000) and rotatory laxity (compared with lateral pivotshift under anesthesia) of the ACL-deficient knee during MRI examination.

Level of evidence Therapeutic studies, Level IV. 
Keywords Knee $\cdot$ Lachman test - Magnetic resonance imaging associated device · Pivot-shift · Rotatory laxity

\section{Introduction}

New technical approaches have been developed to improve the results of single- or double-bundle ACL reconstruction $[17,20]$. Nowadays, patients have higher expectations, and surgeon's goal remains restoring native anatomy and function of the knee and preventing future development of arthritis. However, comparing the results of "standard" ACL single-bundle reconstruction with more anatomic double-bundle reconstruction has proven to be a difficult task [20] and must not rely alone on the subjective clinical evaluation by any manual procedure.

Manual examinations are influenced by surgeon's training [2], and although the pivot-shift test is a better predictor of clinical outcomes as compared to any uniplanar examination procedure, the Lachman test remains the most commonly used $[3,11,12]$.

Nevertheless, the limitations of the pivot-shift test, particularly in an awake patient, must be considered.

In total agreement with the statement of Irrganng et al. [8], the authors firmly believe that a simple clinically applicable tool, similar to the KT-1000 arthrometer can be developed and used to quantify rotational laxity of the knee.

Since the first report [4], the KT-1000 arthrometer (Medmetric, San Diego, CA, USA) is the most widely used knee ligament testing system because it is user-friendly and still remains the reference instrument against which new devices have been tested [26]. However, this is an operatordependent device, which does not measure rotation and has also been associated with false-negative results and questionable reproducibility $[1,10]$.

Thus, the ideal tool to evaluate the knee should be a mean to assess both the "anatomy" and the "function" in the same examination. In order to improve the diagnostic capacity and the way we measure the outcome of ACL reconstruction, a new method was developed using the Porto-knee testing device (PKTD) with measurements performed between bony landmarks.

The purpose of this study is to evaluate the effectiveness of the PKTD to measure antero-posterior and rotatory laxity of the knee during MRI examination. Our hypothesis is that the difference between anterior tibial translations measured in lateral and medial compartment during MRI with PKTD would reflect the results of pivot-shift test in the anesthetized patient.

Thirty-three patients with complaints of unilateral knee instability with suspicion of ACL rupture after clinical evaluation were referred to MRI and randomly selected for evaluation with PKTD at radiology department admission.

\section{Materials and methods}

For the period comprised between 2008 and 2010, 33 patients with complaints of knee instability with signs and symptoms of unilateral ACL rupture were referred to magnetic resonance imaging (MRI) and randomly selected for evaluation with the Porto-knee testing device (PKTD) at radiology department admission. The patients were clinically evaluated at consultation by the senior author (J. E-M). Evaluation was performed using an MRI device (GE Healthcare Signa, USA) operating at 1.5 Tesla field strength in T1- and T2-weighted sequences, including short inversion time inversion recovery (STIR), fat saturation (FatSat) and 3D spoiled gradient recalled (SPGR) reconstruction sequences. PKTD was applied in healthy and injured knees of all patients.

All data from PKTD (including classification of total or partial rupture) were kept confidential until postoperative consultation. Only standard MRI protocol images of injured knee were provided to the surgical team.

Every patient was evaluated preoperatively with KT1000 arthrometer by an investigator independent of surgical team.

A second clinical evaluation, now under anesthesia, was performed by the same surgeon (J. E-M), blinded to PKTD results, according to our standard protocol evaluation for all ACL repairs. This includes the following maneuvers: Lachman test [11] at $25^{\circ}$ flexion; Pivot-shift phenomenon [13] evaluated according to Hughston's Jerk test [7]; anterior drawer at $70^{\circ}$ with foot in neutral, maximal internal and maximal external rotation. Each was graded from 0 to $3+$ using International Knee Document Committee criteria, and then results were registered.

During arthroscopy, ACL ruptures were classified as total, anteromedial (AM)-bundle or posterolateral (PL)bundle ruptures.

Inclusion criteria were as follows: unilateral knee laxity sensation, more than 6 months of lesion and total rupture confirmed during arthroscopy.

Exclusion criteria were previous ACL reconstruction, partial ACL rupture (arthroscopy), and other knee ligaments-associated lesion. Body mass index and meniscus status were also registered.

The study was approved by the Institutional Ethical Committee at the Saúde Atlântica F.C. Porto Sports Center. Standard informed consent was obtained for every patient.

\section{PKTD evaluation protocol}

The PKTD is a knee laxity testing device for measurement of antero-posterior tibial translation and internal rotation of the tibia during the MRI examination (Fig. 1). 

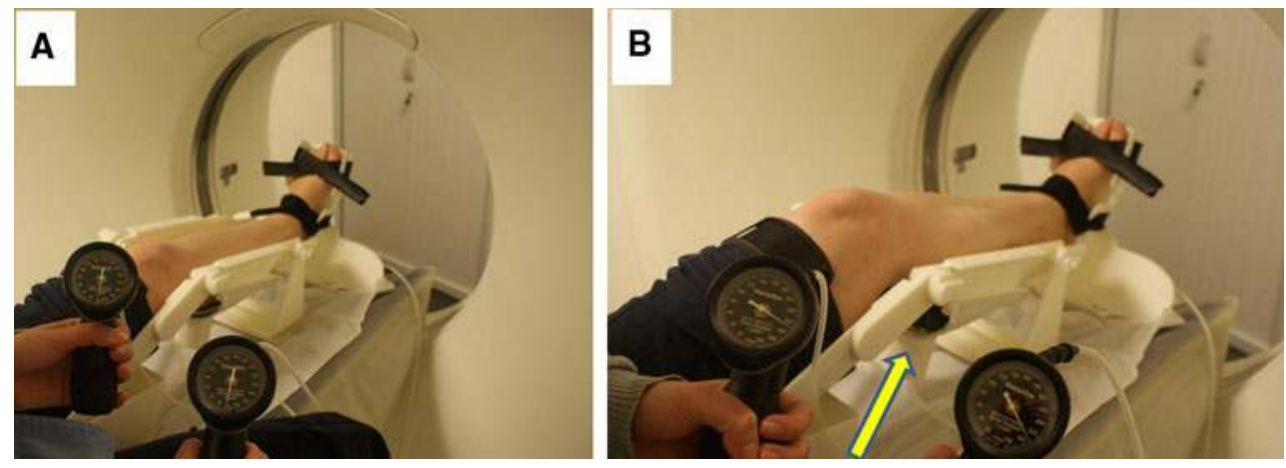

Fig. 1 Photographs of PTKD developed at the Saúde Atlântica F.C. Porto Sports Center: without pressure (a) and with pressure (b). Arrow indicates cuff inducing anterior tibial translation upon pressure application in posterior proximal calf region

PKTD is made of polyurethane, allowing it to be used during MRI scans, in positions in which the knee is placed under stress caused by the inflation of cuffs that have a standardized pressure of $46.7 \mathrm{kPa}$ applied in the posterior proximal calf region, which refers to the load applied per unit area.

Although the PKTD can now be adjusted to different degrees of knee flexion and different degrees of external/ internal rotation inflected by the footplate, all exams herein described were performed at $30^{\circ}$ of flexion in neutral position. The foot rests in the plantigrade position and initial "neutral" rotation; initial foot positioning is determined in each case by dorsiflexion of the ankle (locking effect of talus in ankle mortise); further rotation during pressure application is not restricted. The measurements are determined using two sets of MRI images with $1 \mathrm{~mm}$ spacing, one without (at rest, Fig. 1a) and another with the application of pressure and 3D SPGR sequences (Fig. 1b). Both injured and healthy knees were evaluated.

The measurement (in $\mathrm{mm}$ ) is performed using a line perpendicular to the tibial slope crossing the most posterior point of the tibial plateau and its distance to a parallel line crossing the most posterior point of the femoral condyle as also described by Tashiro et al. [31]. This process is repeated without and with pressure for medial and lateral compartments identifying the same points as bony landmarks. The difference in each of the two points of measurement is calculated between the two sets (without and with pressure) obtaining the anterior translation, in millimeters, for medial and lateral tibial plateaus: MPT, medial plateau translation; LPT, lateral plateau translation (Fig. 2). Similar procedure was performed for healthy and injured knees.

Besides MPT and LPT, the difference between LPT and MPT (LPT - MPT) was calculated. The LPT - MPT reflects the increased translation of lateral tibia condyle associated with rotatory laxity. The registered differences between injured and healthy knees (side-to-side comparison) for MPT, LPT and LPT - MPT were also used as independent variables in order to assess the difference in behavior between stable and ACL-deficient knees (Dif. MPT, Dif. LPT and Dif. LPT - MPT).

\section{Statistical analysis}

The statistical analysis was performed by means of using the commercial SPSS software (IBM SPSS Statistics ver. 20.0; IBM, USA).

The Mann-Whitney $U$ test was used to compare continuous data between the two groups. When the categorical variable has more than two non-ordered groups, the Kruskal-Wallis test was used instead. In order to assess the linear correlation between two variables, the Spearman's rho correlation coefficient was computed along with its significance. When there is no relation between the values of one of the variables, the Pearson correlation was tested for significance.

The Chi-square was used to analyze two categorical variables without order between the categories.

The cut-points were computed maximizing the specificity and sensitivity of the target variable regarding a factor (e.g., Lachman 0 and + against Lachman ++ and +++ ) using the ROC curve coordinates. Statistical significance was set at $p<0.05$.

\section{Results}

From the 33 selected patients, five were excluded from this study after MRI-PKTD evaluation once they were classified as partial ruptures (three as PL-bundle and two as AMbundle ruptures). This classification was confirmed during arthroscopy for all the cases.

For the 28 remaining patients, 3 women and 25 men, with a mean age of $33.4 \pm 9.4$ years, 13 left and 15 right knees were tested. Mean for height was $1.77 \mathrm{~m}$ 

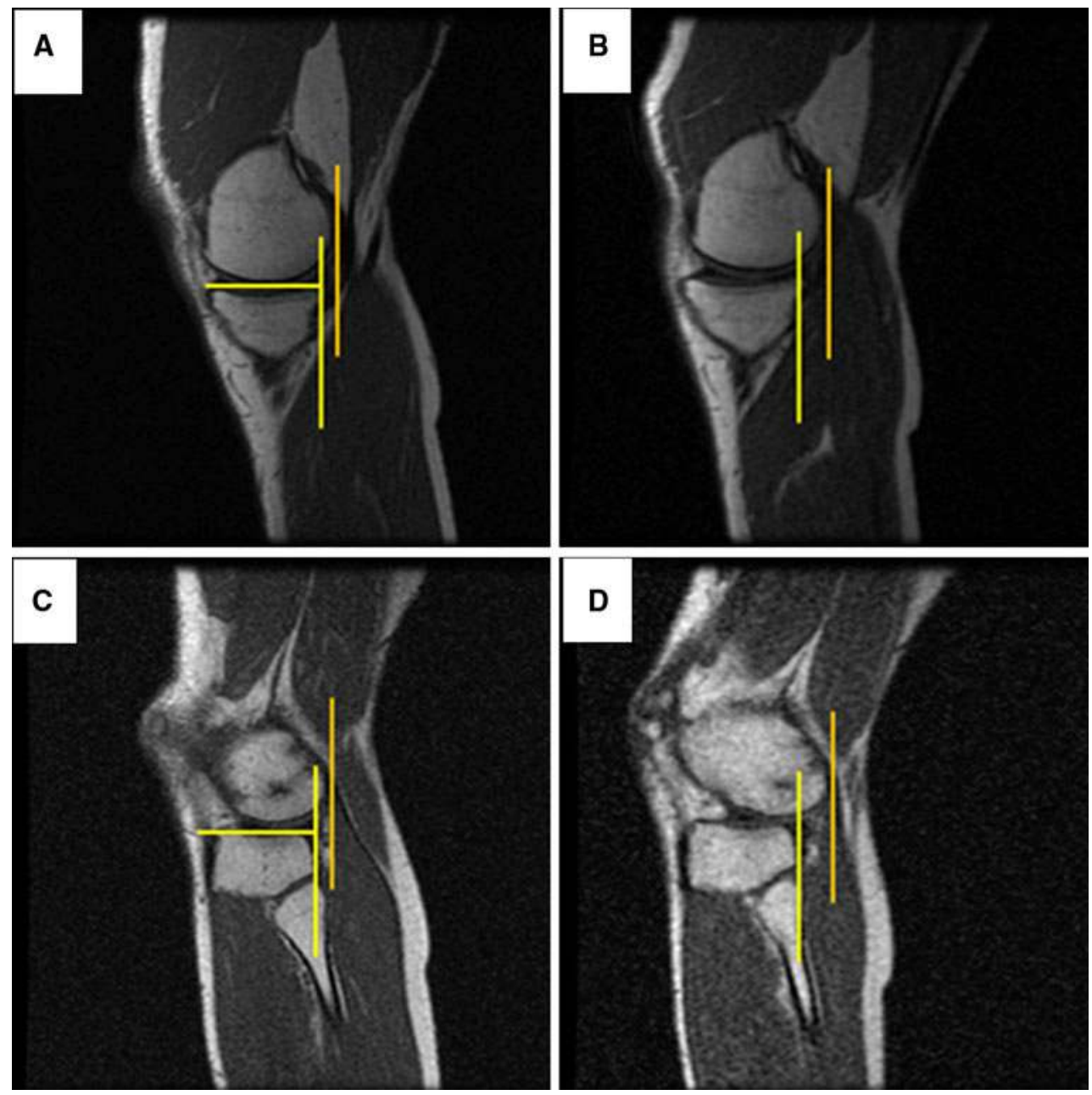

Fig. 2 MRI images of injured knee for medial compartment without (a) and with pressure (b), and for lateral compartment without (c) and with pressure (d), obtained using the PKTD

$(\mathrm{SD}=0.07)$, and for body mass index $(\mathrm{BMI})$, it was 25.3 $(\mathrm{SD}=3.1)$. Isolated lateral meniscus injury was found in 5 $(5 / 28)$, medial meniscus in $10(10 / 28)$ and combined medial and lateral meniscus in 4 (4/28). Figure 3 shows the KT-1000 difference between injured and healthy knees. The mean KT-1000 result for healthy knee was $5.1 \mathrm{~mm}(\mathrm{SD}=0.6)$, and for injured knee, it was $10.5 \mathrm{~mm}(\mathrm{SD}=1.9)$. KT-1000 difference between injured and healthy knees was 3 or higher in all with a mean of $5.4 \mathrm{~mm}(\mathrm{SD}=1.9)$.

The results for clinical examination maneuvers under anesthesia are summarized in Table 1 . Table 2 summarizes the MRI-PKTD evaluation for healthy and injured knees considering medial plateau translation (MPT), lateral plateau translation (LPT) and difference between lateral and medial plateau translations (LPT - MPT). Table 3 presents the registered differences between injured and healthy knees (side-to-side comparison) for MPT, LPT and LPT - MPT. Table 4 summarizes the correlation between

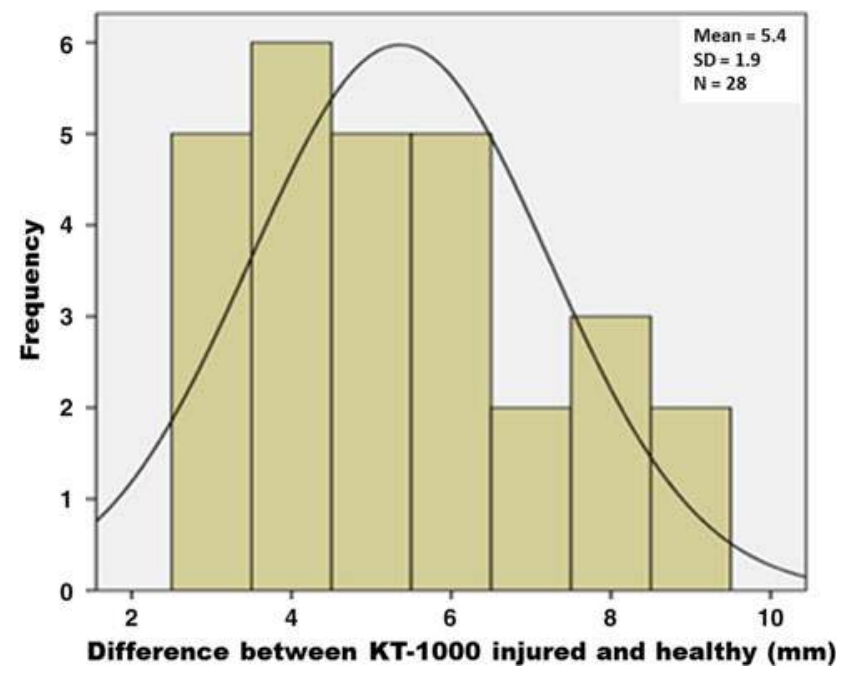

Fig. 3 KT-1000 evaluation of the differences between injured and healthy knees 
Table 1 Results achieved by manual tests (clinical evaluation under anesthesia)

\begin{tabular}{lrrrr}
\hline Manual test & 0 & + & ++ & +++ \\
\hline Lachman (mm) & 0 & 2 & 10 & 16 \\
Pivot-shift (mm) & 5 & 3 & 11 & 9 \\
AD Neutral (mm) & 0 & 4 & 18 & 6 \\
AD Int. Rot. (mm) & 4 & 21 & 3 & 0 \\
AD Ext. Rot. (mm) & 0 & 6 & 17 & 5 \\
\hline
\end{tabular}

Table 2 Results achieved by MRI-PKTD evaluation of healthy and injured knees

\begin{tabular}{lllll}
\hline PKTD & $\begin{array}{l}\text { Minimum } \\
(\mathrm{mm})\end{array}$ & $\begin{array}{l}\text { Maximum } \\
(\mathrm{mm})\end{array}$ & $\begin{array}{l}\text { Mean } \\
(\mathrm{mm})\end{array}$ & SD \\
\hline $\begin{array}{l}\text { Healthy knee } \\
\text { MPT }\end{array}$ & 1 & 3 & 1.7 & 0.6 \\
LPT & 1 & 3 & 1.7 & 0.6 \\
LPT - MPT & 0 & 1 & 0.3 & 0.5 \\
Injured knee & & 14 & & \\
MPT & 5 & 10 & 10.1 & 2.6 \\
LPT & 4 & 8 & 6.5 & 1.5 \\
LPT - MPT & 1 & 4.5 & 2.0 \\
\hline
\end{tabular}

$\overline{M P T}$ medial plateau translation, $L P T$ lateral plateau translation, $L P T-$ $M P T$ difference between lateral and medial plateau translation

Table 3 Results achieved by PKTD evaluation of injured - healthy knees

\begin{tabular}{llcll}
\hline $\begin{array}{l}\text { PKTD injured }- \\
\text { healthy knees }\end{array}$ & $\begin{array}{l}\text { Minimum } \\
(\mathrm{mm})\end{array}$ & $\begin{array}{l}\text { Maximum } \\
(\mathrm{mm})\end{array}$ & $\begin{array}{l}\text { Mean } \\
(\mathrm{mm})\end{array}$ & SD \\
\hline Dif. MPT & 1 & 8 & 4.2 & 1.6 \\
Dif. LPT & 4 & 12 & 8.4 & 2.2 \\
Dif. LPT - MPT & 1 & 8 & 4.2 & 1.9 \\
\hline
\end{tabular}

MPT, LPT and LPT - MPT were used as independent variables to determine the difference in behavior between stable and ACL-deficient knees (Dif. MPT, Dif. LPT and Dif. LPT - MPT) the different tests. We were not able to establish statistically significant correlation between Lachman test results and Dif. LPT - MPT, LPT - MPT, either for Dif. MPT or for Dif. LPT (n.s.). For lateral pivot-shift test, a strong significant correlation was established for LPT - MPT, Dif. LPT MPT and Dif. LPT. The threshold level for a grade 2 or higher pivot-shift test in this study is $8.5 \mathrm{~mm}$ for Dif. LPT, and $3.5 \mathrm{~mm}$ for LPT - MPT and Dif. LPT - MPT.

A positive and significant correlation was also found for anterior drawer test in external rotation (AD Ext. Rot.) and Dif. LPT - MPT (cor. coefficient $=0.38 ; p<0.05$ ). The threshold level based on AD Ext. Rot. $(0 / 1+$ vs. $2+/ 3+)$ for Dif. LPT - MPT is $4.5 \mathrm{~mm}$. Similar analysis demonstrated absence of significant correlation for all other manual tests.

Considering the KT-1000 difference between injured and healthy knees, a very strong positive correlation was found for Dif. MPT and Dif. LPT, but not for Dif. LPT - MPT (cor. coefficient $=0.08$ ) (n.s.). The threshold value-based KT-1000 difference between injured and healthy knees (0-5 vs. $\geq 6$ ) for Dif. MPT is $4.5 \mathrm{~mm}$, and for Dif. LPT, it is $7.5 \mathrm{~mm}$.

Body mass index had a negative correlation with Dif. LPT - MPT, but no other significant correlation with PKTD values, manual tests or KT-1000 evaluation was found. Statistical significance was obtained for Dif. LPT - MPT and weight, but not for height (n.s.).

Gender did not statistically influence Lachman or pivotshift test (Pearson test; n.s.). By its turn, gender also has no influence on KT-1000 or Dif. LPT - MPT (Mann-Whitney test; n.s.). No significant influence of associated meniscus injury could be established for manual tests, KT-1000 or PKTD results (n.s.).

\section{Discussion}

The most important finding of the present study is that the PKTD proved to be as reliable as KT-1000 in assessing antero-posterior translation. The PKTD also showed to be as feasible as lateral pivot-shift for quantification of
Table 4 Results of the correlation coefficient achieved for the different tests

\begin{tabular}{lllll}
\hline & $\begin{array}{l}\text { Dif. LPT }- \text { MPT } \\
\text { (cor. coefficient; } \\
p \text { value }\end{array}$ & $\begin{array}{l}\text { LPT }- \text { MPT } \\
\text { (cor. coefficient; } \\
p \text { value })\end{array}$ & $\begin{array}{l}\text { Dif. MPT } \\
\text { (cor. coefficient; } \\
p \text { value) }\end{array}$ & $\begin{array}{l}\text { Dif. LPT } \\
\text { (cor. coefficient; } \\
p \text { value) }\end{array}$ \\
\hline $\begin{array}{l}\text { Lachman test } \\
\text { Pivot-shift }\end{array}$ & $0.23 ;$ n.s. & $0.32 ;$ n.s. & $-0.18 ;$ n.s. & $0.10 ;$ n.s. \\
$\begin{array}{l}\text { KT-1000 difference } \\
\text { between injured and } \\
\text { healthy knees }\end{array}$ & $0.03 ;<0.05$ & $0.80 ;<0.05$ & $-0.34 ;$ n.s. & $0.43 ;<0.05$ \\
$\begin{array}{l}\text { Body mass index } \\
\text { Weight }\end{array}$ & $-0.38 ;<0.05$ & - & $0.73 ;<0.05$ & $0.5 ;<0.05$ \\
Height & $-0.47 ;<0.05$ & - & - & - \\
\hline
\end{tabular}


rotation (evaluation under anesthesia). A limitation of static laxity measurement devices is the unknown starting position of rotation. This is also a drawback to consider in the present study since starting position was based on ankle mortise, a maneuver that cannot be considered precise or reproducible. However, the advantage of PKTD is the ability to evaluate the knee at any angle of rotation between internal maximum and external maximum. Future study is planned to compare results of foot in neutral without rotational restriction (as herein reported) with forced maximum external and internal rotation during anteroposterior load transmission using the PKTD.

The patients were clinically evaluated by a single examiner, and inherent bias might be considered. However, this reflects the standard operating procedure established within our group (all cases are evaluated under anesthesia by responsible surgeon). This protocol was chosen to minimize bias throughout the study.

Okazaki et al. [23] quantified the anterolateral rotational laxity of ACL-deficient knees by pivot-shift test in open MRI. Using the same method, Tashiro et al. [31] demonstrated that side-to-side differences in anterolateral tibial translation correlates with clinical grade of pivot-shift test and stress radiography, but not with KT-2000 arthrometry $(n=20)$. The threshold value was established as $3.0 \mathrm{~mm}$, which can be considered in line with the results herein described (threshold level for Dif. LPT - MPT is $3.5 \mathrm{~mm}$ ). Using also the aforementioned method, Izawa et al. [9] reported better rotatory stability of anatomic double-bundle reconstruction as compared to single-bundle reconstruction. However, open MRI devices are not routinely available in most knee surgery centers, and despite that intra- and inter-observer reproducibility have been reported, the method requires availability of a trained surgeon capable of executing pivot-shift test during MRI. On the other hand, it has also been demonstrated that mechanized pivot-shift achieves greater accuracy than manual testing [21].

Several other methods have been proposed [2, 25] aiming to quantify rotational laxity in an objective and reliable method including robotics [34], navigation (computer-assisted) [35], radiostereometric analysis [30], stress radiographies [27] or several arthrometers [2, 3, 14, 15, 19, $24,26,32]$ reflecting the growing consensus around the need to describe objectively the rotational behavior of knee joint for diagnostic purposes, detecting risk factors or controlling surgical outcomes.

Our study failed to demonstrate significant correlation between Lachman test and the difference in tibial translation registered between lateral and medial compartment comparing side-to-side or considering the injured knee alone. This reinforces that Lachman test, considered for long time the benchmark to assess success in ACL repair, is not suitable for the present clinical needs.
Concerning the lateral pivot-shift test, a strong positive correlation was noticed between a higher grade and the difference between medial and lateral tibial translation of ACL-deficient knees (threshold level for $2+/ 3+$ is $3.5 \mathrm{~mm}$ ). Because of this positive correlation, rotatory laxity can be assessed utilizing the PKTD. Considering side-to-side differences, similar correlations were achieved for the difference in lateral translation and differential between lateral and medial translations. Surprisingly, a negative correlation near statistical significance was noticed for side-to-side difference in medial compartment anterior displacement. This might reflect that some restriction in freedom of motion in medial compartment increases the pivot-shift phenomenon and thus should be addressed in future investigation.

For KT-1000 side-to-side difference, a strong positive correlation was registered for side-to-side difference in the amount of translation either in medial or in lateral compartment. However, if the difference in translation in lateral and medial compartments (which somewhat reflects rotation) is considered, the results are not statistical significant. This is also in line with the concept that KT-1000 is a valuable tool to assess antero-posterior laxity, but not rotation.

Neither gender nor meniscus injury demonstrated influence in results of clinical maneuvers, KT-1000 or PKTD. Although, sample size and study design do not allow us to reach further conclusions with respect to this subject.

The inverse correlation of BMI and weight with side-toside comparison of rotation (Dif. LPT - MPT) highlights the fact that higher weight and BMI index might increase difficulties in assessment of lateral pivot-shift either manually or using mechanized methods. This issue should be considered for subsequent improvements of devices. One should not only standardize and control load transmission, but probably also adapt it accordingly.

Partial ACL ruptures [22, 29], which are difficult to recognize by preoperative MRI [33], are of great interest. Despite being considered out of the scope of this study, the five cases in our initial group identified with isolated AM or PL bundle were correctly identified (in concordance with arthroscopic findings) using MRI with PKTD. Its unique feature of combining anatomical and dynamic evaluation might improve the effectiveness of radiologists to detect and evaluate partial lesions in MRI. By means of inducing ACL tension during the exam, it is possible not only to observe the mechanical behavior of partial ruptures $[16,28]$, but also to enhance the visualization of "biologic"/signal characteristics of the ruptured and remaining bundle. This important aspect requires further investigation.

PKTD might also play a future role in prevention (detecting risk factors) and/or identifying those patients 
presenting higher rotatory laxity and those who may require an ACL reconstruction technique, which provides higher rotational constraint (e.g., double bundle) [6].

These data provide one further step in understanding knee kinematics, but their functional implication and the way in which they might affect ACL reconstruction are not fully achieved. ACL research demands perseverance and patience [18] although being an exciting and rewarding field. Recognizing that the application of combined internal rotation and valgus torques to the knee can more precisely recreate the anterolateral subluxation that occurs in knee joint during the pivot-shift test [5], we do believe that foot rotation should not be restricted while applying translational force.

\section{Conclusion}

The PKTD is a useful, reliable device to quantify anteroposterior and rotatory laxity of the knee. MRI-PKTD evaluation proved to be reliable in the assessment of antero-posterior translation (comparing with KT-1000) and rotatory laxity (compared with lateral pivot-shift under anesthesia) of the ACL-deficient knee.

It might play a future role in prevention, indications for surgical versus conservative treatment, identifying partial ruptures and the status of the remaining bundle due to its unique feature of combining anatomical and dynamic "clinical" evaluation amplified by the high-resolution medical MRI.

Acknowledgments The authors wish to thank Pedro Campelos, André Silva and Rogério Pereira for their clinical and technical support in the field of research at Saúde Atlântica F.C. Porto Sports Center.

\section{References}

1. Boyer P, Djian P, Christel P, Paoletti X, Degeorges R (2004) Reliability of the KT-1000 arthrometer (Medmetric) for measuring anterior knee laxity: comparison with Telos in 147 knees. Rev Chir Orthop Reparatrice Appar Mot 90(8):757-764

2. Branch TP, Mayr HO, Browne JE, Campbell JC, Stoehr A, Jacobs CA (2010) Instrumented examination of anterior cruciate ligament injuries: minimizing flaws of the manual clinical examination. Arthroscopy 26(7):997-1004

3. Citak M, Suero EM, Rozell JC, Bosscher MR, Kuestermeyer J, Pearle AD (2011) A mechanized and standardized pivot shifter: technical description and first evaluation. Knee Surg Sports Traumatol Arthrosc 19(5):707-711

4. Daniel DM, Stone ML, Sachs R, Malcom L (1985) Instrumented measurement of anterior knee laxity in patients with acute anterior cruciate ligament disruption. Am J Sports Med 13(6):401-407

5. Engebretsen L, Wijdicks CA, Anderson CJ, Westerhaus B, Laprade RF (2011) Evaluation of a simulated pivot shift test: a biomechanical study. Knee Surg Sports Traumatol Arthrosc. doi: 10.1007/s00167-011-1744-1

6. Hemmerich A, van der Merwe W, Batterham M, Vaughan CL (2011) Knee rotational laxity in a randomized comparison of single- versus double-bundle anterior cruciate ligament reconstruction. Am J Sports Med 39(1):48-56

7. Hughston JC, Andrews JR, Cross MJ, Moschi A (1976) Classification of knee ligament instabilities. Part I. The medial compartment and cruciate ligaments. J Bone Joint Surg Am 58(2):159-172

8. Irrgang JJ, Bost JE, Fu FH (2009) Re: Outcome of single-bundle versus double-bundle reconstruction of the anterior cruciate ligament: a meta-analysis. Am J Sports Med 37(2):421-422

9. Izawa T, Okazaki K, Tashiro Y, Matsubara H, Miura H, Matsuda S, Hashizume M, Iwamoto Y (2011) Comparison of rotatory stability after anterior cruciate ligament reconstruction between single-bundle and double-bundle techniques. Am J Sports Med 39(7):1470-1477

10. Jardin C, Chantelot C, Migaud H, Gougeon F, Debroucker MJ, Duquennoy A (1999) Reliability of the KT-1000 arthrometer in measuring anterior laxity of the knee: comparative analysis with Telos of 48 reconstructions of the anterior cruciate ligament and intra- and interobserver reproducibility. Rev Chir Orthop Reparatrice Appar Mot 85(7):698-707

11. Katz JW, Fingeroth RJ (1986) The diagnostic accuracy of ruptures of the anterior cruciate ligament comparing the Lachman test, the anterior drawer sign, and the pivot shift test in acute and chronic knee injuries. Am J Sports Med 14(1):88-91

12. Kocher MS, Steadman JR, Briggs KK, Sterett WI, Hawkins RJ (2004) Relationships between objective assessment of ligament stability and subjective assessment of symptoms and function after anterior cruciate ligament reconstruction. Am J Sports Med 32(3):629-634

13. Lane CG, Warren R, Pearle AD (2008) The pivot shift. J Am Acad Orthop Surg 16(12):679-688

14. Lob T, Verheyden AP, Josten Ch, F S (2006) The function of the ACL measured in an vertical opened MRI (0.5 Tesla). Paper presented at the 12th ESSKA Congress, Innsbruck, Austria

15. Lorbach O, Wilmes P, Theisen D, Brockmeyer M, Maas S, Kohn D, Seil R (2009) Reliability testing of a new device to measure tibial rotation. Knee Surg Sports Traumatol Arthrosc 17(8):920-926

16. Lorenz S, Illingworth KD, Fu FH (2009) Diagnosis of isolated posterolateral bundle tears of the anterior cruciate ligament. Arthroscopy 25(11):1203-1204

17. Lubowitz JH, Ahmad CS, Anderson K (2011) All-inside anterior cruciate ligament graft-link technique: second-generation, noincision anterior cruciate ligament reconstruction. Arthroscopy 27(5):717-727

18. Lubowitz JH, Poehling GG (2010) Understanding ACL research requires patience and persistence. Arthroscopy 26(7):869-871

19. Mayr HO, Hoell A, Bernstein A, Hube R, Zeiler C, Kalteis T, Suedkamp NP, Stoehr A (2011) Validation of a measurement device for instrumented quantification of anterior translation and rotational assessment of the knee. Arthroscopy 27(8):1096-1104

20. Meredick RB, Vance KJ, Appleby D, Lubowitz JH (2008) Outcome of single-bundle versus double-bundle reconstruction of the anterior cruciate ligament: a meta-analysis. Am J Sports Med 36(7):1414-1421

21. Musahl V, Voos J, O’Loughlin PF, Stueber V, Kendoff D, Pearle AD (2010) Mechanized pivot shift test achieves greater accuracy than manual pivot shift test. Knee Surg Sports Traumatol Arthrosc 18(9):1208-1213

22. Ochi M, Adachi N, Deie M, Kanaya A (2006) Anterior cruciate ligament augmentation procedure with a 1-incision technique: 
anteromedial bundle or posterolateral bundle reconstruction. Arthroscopy 22(4):e461-e465

23. Okazaki K, Miura H, Matsuda S, Yasunaga T, Nakashima H, Konishi K, Iwamoto Y, Hashizume M (2007) Assessment of anterolateral rotatory instability in the anterior cruciate ligamentdeficient knee using an open magnetic resonance imaging system. Am J Sports Med 35(7):1091-1097

24. Pollet V, Barrat D, Meirhaeghe E, Vaes P, Handelberg F (2005) The role of the Rolimeter in quantifying knee instability compared to the functional outcome of ACL-reconstructed versus conservatively-treated knees. Knee Surg Sports Traumatol Arthrosc 13(1):12-18

25. Pugh L, Mascarenhas R, Arneja S, Chin PY, Leith JM (2009) Current concepts in instrumented knee-laxity testing. Am J Sports Med 37(1):199-210

26. Robert H, Nouveau S, Gageot S, Gagniere B (2009) A new knee arthrometer, the GNRB: experience in ACL complete and partial tears. Orthop Traumatol Surg Res 95(3):171-176

27. Schulz MS, Russe K, Lampakis G, Strobel MJ (2005) Reliability of stress radiography for evaluation of posterior knee laxity. Am J Sports Med 33(4):502-506

28. Sonnery-Cottet B, Chambat P (2007) Arthroscopic identification of the anterior cruciate ligament posterolateral bundle: the figureof-four position. Arthroscopy 23(10):e1121-e1123

29. Sonnery-Cottet B, Lavoie F, Ogassawara R, Scussiato RG, Kidder JF, Chambat P (2010) Selective anteromedial bundle reconstruction in partial ACL tears: a series of 36 patients with mean
24 months follow-up. Knee Surg Sports Traumatol Arthrosc 18(1):47-51

30. Sorensen OG, Larsen K, Jakobsen BW, Kold S, Hansen TB, Lind M, Soballe K (2011) The combination of radiostereometric analysis and the telos stress device results in poor precision for knee laxity measurements after anterior cruciate ligament reconstruction. Knee Surg Sports Traumatol Arthrosc 19(3):355-362

31. Tashiro Y, Okazaki K, Miura H, Matsuda S, Yasunaga T, Hashizume M, Nakanishi Y, Iwamoto Y (2009) Quantitative assessment of rotatory instability after anterior cruciate ligament reconstruction. Am J Sports Med 37(5):909-916

32. Tsai AG, Musahl V, Steckel H, Bell KM, Zantop T, Irrgang JJ, Fu FH (2008) Rotational knee laxity: reliability of a simple measurement device in vivo. BMC Musculoskelet Disord 9:35-43

33. Van Dyck P, De Smet E, Veryser J, Lambrecht V, Gielen JL, Vanhoenacker FM, Dossche L, Parizel PM (2011) Partial tear of the anterior cruciate ligament of the knee: injury patterns on MR imaging. Knee Surg Sports Traumatol Arthrosc. doi: 10.1007/s00167-011-1617-7

34. Woo SLY, Fisher MB (2009) Evaluation of knee stability with use of a robotic system. J Bone Joint Surg (Am) 91(1):78-84

35. Zaffagnini S, Bignozzi S, Martelli S, Imakiire $\mathrm{N}$, Lopomo $\mathrm{N}$, Marcacci M (2006) New intraoperative protocol for kinematic evaluation of ACL reconstruction: preliminary results. Knee Surg Sports Traumatol Arthrosc 14(9):811-816 